$L A--9051$

DE82 007034

\section{LA-9051}

UC.34c

Issued: December 1981

\title{
On Photoneutron Generation with Electron Undulators
}

\author{
G. C. Baldwin
}

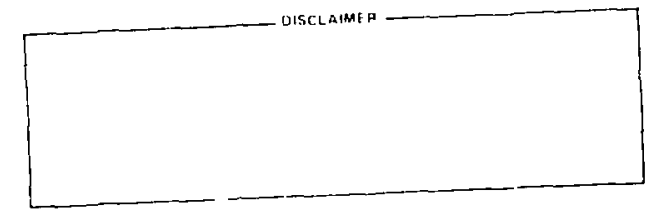




\title{
ON PHOTONEUTRON GENERATION WITH ELECTRON UNDULATORS
}

by

G. C. Baldwin

\begin{abstract}
This report proposes an extremely compact line-geometry pulsed source of photoneutrons, with controllable energies of only a few kiloelectron volts, average yields exceeding 1(13) per second, peak intensities higher than the average by two or three orders of magnitude, controllable polarization, and pulse durations of about $2 \mathrm{~ns}$. This source uses photons from a magnetic undulator in a $30-\mathrm{GeV}$ electron-storage ring. Parameters of the undulator and storage ring are stringent but probably attainable with careful design and construction. Several applicutions are discussed.
\end{abstract}

\section{INTRODUCTION}

An intense source of polarized neutrons with energies in the resonance region; of relatively small dimensions: and with yield, energy, and bandwidth at the control of the experimenter, would have scientific and technological importance. Therefore. serious attention is warranted for recent suggestions ${ }^{1.2}$ to extend the energy of the so-calied synchrotron radiation ${ }^{3 \cdot 10}$ above the neutron binding energy of ${ }^{9} \mathrm{Be}(1665 \mathrm{keV}) .{ }^{2} \mathrm{D}(2223 \mathrm{keV})$, or even heavier nuclei (6-20 MeV). thereby creating an intense source of photoneutrons.

Although photoneutron sources using radioisotopes were first developed 50 years ago, they continue to find research applications because they gerierate relatively sharp line spectra of neutrons in the resonance region. between thermal and a few tens of keV. Unfortunately. only a few energies are available. the yields are weak. and the source must be frequently reactivated. All other types of neutron sources generate fast neutrons. which usually must be moderated before they can be used.

Research on interactions of neutrons at other energies in the resonance region requires pulsed accelerator or reactor sources using time-of-night (TOF) or angular dependence for energy selection. The resolution of the
TOF method is limited by fluctuations in moderation time and by the finite dimensions of the neutron mod. crator.

Proposals to form Mossbauer isomers in high concen tration to pump gamma-ray lasers (grasers) by neutron capture ${ }^{12.11}$ are frustrated also by the need to moderate neutrons. The spread of moderation times exceeds decay lifetimes of most Mössbauer isomers and, owing to moderator heating. neutrons cannot be slowed down for capturing in the densities required to establish population inversion if they have been generated by fission. fusion. or spallation.

\section{PROPOSED USE OF SYNCHROTRON RADI- ATION FOR NEUTRON GENERATION}

In a brief paper. Eremeev' suggests that synchrotron radiation can be made sufliciently intense and energetic to generate high yields of photoneutrons from an extremely small volume of beryllium. Table I, taken from his article, compares numbers of neutrons that might he generated with radiation from the $7 . \mathrm{GeV}$ electron-storage ring at Novosibirsk, USSR, with those fium 


\begin{tabular}{|c|c|c|c|}
\hline \multirow[b]{2}{*}{ Parameters } & \multicolumn{2}{|c|}{$\begin{array}{c}\text { NF, VEPP-4 GeV Be }(\gamma, 11), \\
E_{B}=7 \mathrm{GeV}\end{array}$} & \multirow{2}{*}{$\begin{array}{c}\text { Linear electron amplifier } \\
\begin{array}{c}\mathrm{U}(\beta \rightarrow \gamma, \mathrm{n}) \\
v=900 \mathrm{~Hz}\end{array} \\
\mathrm{H}_{2} \mathrm{O}=\text { Moderator. }\end{array}$} \\
\hline & $\begin{array}{c}v=10^{6} \mathrm{~Hz} \\
<\mathrm{H}>=60 \mathrm{kOe}\end{array}$ & $\begin{array}{c}v=20 \mathrm{~Hz} \\
\mathrm{H} \approx 250 \mathrm{kOe}\end{array}$ & \\
\hline Pulse duration, s & $10^{t 0}$ & $10^{-10}$ & $10^{-7}$ \\
\hline Intensity, $\mathrm{n} / \mathrm{s}$ & $3 \times 10^{16}$ & $3.5 \times 10^{20}$ & $2 \times 10^{16}$ \\
\hline$F l_{\mathrm{L} n}, n / \mathrm{cm}^{2}$ & $2.5 \cdot 10^{15}$ & $3 \times 10^{19}$ & $3.5 \times 10^{13}$ \\
\hline Gen. density, $\mathrm{n} / \mathrm{cm}^{3}$ & $1 \cdot 10^{17}$ & $1 \times 10^{21}$ & $2.5 \times 10^{13}$ \\
\hline Intensity, $\mathrm{n} / \mathrm{s}$ & $3 \times 10^{12}$ & $2 \times 10^{12}$ & $2 \times 10^{12}$ \\
\hline Flux, $\mathrm{n} / \mathrm{cm}^{2} \mathrm{~s}$ & $2.5 \times 10^{11}$ & $1.5 \times 10^{11}$ & $3 \times 10^{9}$ \\
\hline Gen. density, $n / \mathrm{cm}^{3} \mathbf{s}$ & $1 \times 10^{13}$ & $6 \times 10^{12}$ & $2.5 \times 10^{9}$ \\
\hline Power in target, $\mathrm{kW}$ & $0.3^{\sharp}$ & 0.3 & 4.0 \\
\hline Quality, $n / s$ & $3 \cdot 10^{32}$ & $2 \times 10^{32}$ & $2 \times 10^{26}$ \\
\hline Effective gen. volume, $\mathrm{cm}^{3}$ & 0.3 & 0.3 & $785^{h}$ \\
\hline \multirow[t]{2}{*}{ Radiation losses in target, $\mathrm{MeV} / \mathrm{n}$} & 450 & 450 & $1250^{c}$ \\
\hline & & & $13000^{d}$ \\
\hline $\begin{array}{l}\text { 'With allowance for filtering the synch } \\
\text { "Moderator volume, thickness } 3 \mathrm{~cm} \text {. } \\
\text { 'Per neutron produced in the target. } \\
\text { dPer neutron moderated to an energy }\end{array}$ & 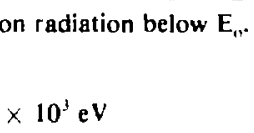 & & \\
\hline
\end{tabular}

a typical accelerator source. He estimates average neu tron yields of the order of $2(12)^{*} \mathrm{~s}$ ' in pulses of $0.1 \mathrm{~ns}$ duration: effective source volume is $0.3 \mathrm{~cm}^{3}$ and only a few hundred watts of power would be dissipated. By increasing the beam energy to $15.20 \mathrm{GeV}$ and using pulsed magnetic fields of $25-\mathrm{T}$ incuction. Eremeev claims that $1(20) \mathrm{n} \mathrm{cm}^{2} s^{\prime}$ in a fat energy spectrum extending from 0 to $250 \mathrm{keV}$ can be achieved. According to Eremeev. neutrons from such a source could excite nuclei, perhaps pump a graser, scudy coherent phenomena in the interactions of neutrons in solids, and even investigate the neutron-neutron interaction. His article does not give enough information to permit detailed verification of these assertions, however.

\footnotetext{
"Powers of ten are given in the "floating point" notation; thus
} a.bc...(d) denotes a.bc... $\times 10^{d}$.
Eremeev's estimates are based on the asymptotic form of the spectrum of synchrotron radiation ${ }^{14}$ above its critical energy: that is, on the radiation from electrons orbiting in a bending magnet. He assumes that the angular distribution of the radiation is that from a single clectron. and he neglects the intrinsic spatial and angular spread of electrons in the beam. His beryllium target would be isradiated and. therefore. heated by the entire continuous spectrum of this radiation, only a small part of which would be above the ${ }^{9} \mathrm{Be}(\gamma, \pi)$ reaction threshold.

Eremeev's approach seems less promising than one using a magnetic undulator ${ }^{2.15,17}$ to generate a line spectrum of photons above the $(y, n)$ threshold. Undulators can generate up to four orders of magnitude higher spectral brightness from a given electron bcam than can bending magnets, in spectral lines so narrow that approximately monoenergetic photons eventually 
may be possible. The intensity of background radiation is then far less than in the case of synchrotron radiation.

This prospect is not widely recognized, although several groups are considering synchrotron radiation sources for photonuclear research at higher energies. In this report, we describe the characteristics of several existing and planned electron-storage rings, give formulas for undulator radiation, summarize information required for estimating neutron yields and spectra from beryllium, estimate neutron yield from an ideal source, suggest possible applications, and point out challenges this concept presents to accelerator design.

\section{ESSENTIAL ELEMENTS OF AN ELEC TRON-UNDULATOR PHOTONEUTRON SOURCE}

In this system, the electron-storage ring comprises an evacuated path for electrons guided in a closed orbit by a large number of bending and focussing magnets, together with rf cavities that apply longitudinal electric fields synchronously with the passage of e'ectron bunches, to compensate for radiation losses of the electrons (Fig. 1).

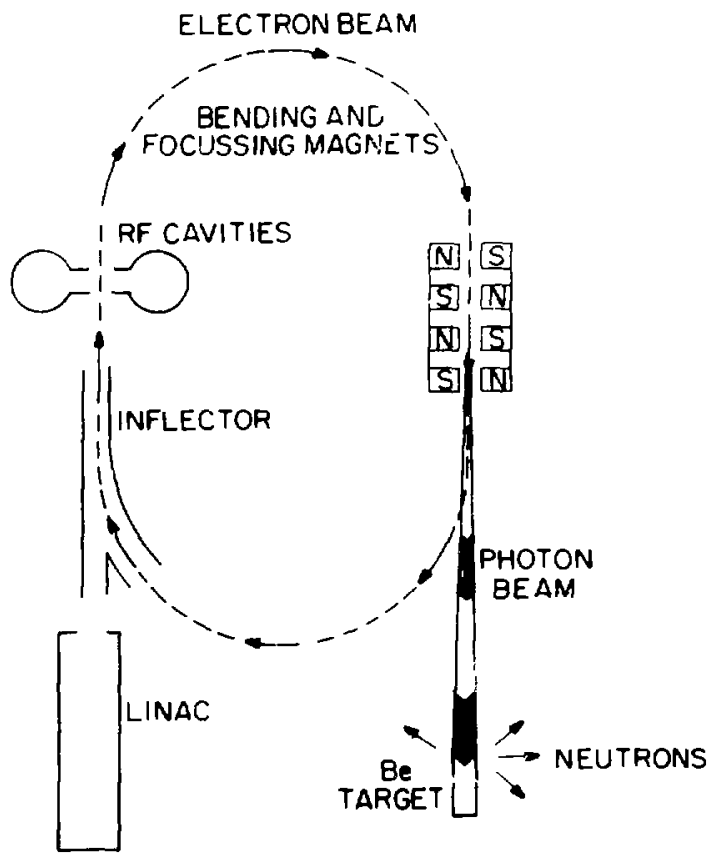

Fig. 1. Essential elements of an undulator photoneutron source (UrS). The electron beam is obtained by occasional injection from an external accelerator. here shown as a linac.
The storage ring contains several straight sections: one that accommodates an inflection system that can inject relativistic electrons from a high-energy accelerator at intervals of several hours to replenish the beam in the stcrage ring: and others that may contain unduiators or other apparatus. Neutrons ar generated in a beryllium target by the emitted radiation when the undulator and beam parameters are properly adjusted. Although storage rings were originally developed for colliding-beam, high-energy physics research, today dedicated facilities are available for production of ultraviolet and $x$-radiation. ${ }^{3 \cdot 10}$

An undulator ${ }^{19-17}$ comprises a set of $2 \mathrm{~N}$ dipole magnets that establish fields transverse* to the electron-beam-axis. alternated in direction. The field causes the electrons to oscillate transversely to the beam-axis. Each electron in the undulator behaves as a moving sipole radiator that. at relativistic energies, emits photons into a narrow cone in the forward direction. concentric with the beam axis.

The theory of undulator radiation is discussed in many references: ${ }^{15} 17$ essential formulas are given in Sec. VI. Here we note that the spatial period of the magnetic field in the undulator $\lambda_{u}$ appears Lorentz-contracted to the moving electrons, so that the $v \times B$ force they experience alternates in direction at a frequency that, in a system moving with the electrons at their averam axial velocity Bc. is given by

$\omega_{0}=\gamma \frac{\mathrm{c}}{i_{u}}$

where

$\gamma=\left(1-\beta^{2}\right)^{1 \cdot 2}$.

Photons radiated by the oscillating electron are seen, in a reference frame moving with its average axial velocity. to have this same frequency. However, to a laboratory observer, this radiation is Doppler-shifted. introducing a second factor of $\gamma$. so that the fundamental

- Helical undulators ${ }^{18,14}$ have been used with linear ac. celerators (linacs). Their use might be difficult for storage rings because they would interfere with injection into the ring. This perhaps could be avoided by "switching" the beam into the undulator after the main ring had been loaded, using a fast pulsed bending magnet. Helical undulators generate circularly polarized gamma radiation. 
wavelength of the undulator radiation in the laboratory frame is of the order of

$\lambda_{1} \sim \frac{\lambda_{\mathrm{u}}}{\gamma^{2}}$

Higher harmonics also are generated under conditions described in Sec. XII. Their bandwidths are inversely proportional to the number of oscillations undergone in transit through the undulator; that is, to the product of the number $\mathrm{N}$ of undulator field reversals by the order $\mathrm{m}$ of the harmonic. This radiation is contained within the light-cone of the relativistic electron source; that is, within an angie of about $\gamma^{-1}$ rad. Thus, a well-collimated beam of high-energy photons, linearly* polarized and relatively monochromatic, is emitted within the vacuum enclosure into the forward direction.

An alternative analysis considers tine Lorentz-transformed undulator field, as seen from an observer moving with the electrons, to be an approaching electromagnetic wave, from which photons are scattered by electrons. In the back-scattered case, the Doppler effect increases the energy of the scattered photons into the range given by Eq. (3). If the values of $\lambda_{u}^{-1}$ and $\gamma$ are high enough. these photons can undergo the $(\gamma, n)$ reaction in appropriate targets. We estimate the photon intensity in Sec. VI and the neutron yields for beryllium in Sec. VII.

The highly collimated undulator radiation interacts at the target within an elongated segment of a very narrow cone. Scattering and absorption in this region almost invariably should result in escape of the secondary radiations out the sides. Therefore, although attenuation of the photon beam is representable oy the total nar. row-beam cross section. the energy transferred to the target medium is far less than that found by using the energy-absorption cross section, if the target is in the ideal furm of a narrow filament concentric with the beam.

\section{ELECTRON-STORAGE-RING PARAMETERS}

For our purposes, the important parameters are the energy of the circulating electrons $E_{0}(\mathrm{GeV})$ and the corresponding relativistic factor.

$\gamma=1957 \mathrm{E}_{\mathbf{o}}$;

- Or circularly, if a helical undulator can be used with the storage ring. the circulating beam current I (A); the effective radius of curvature of the electron orbit in the main storage ring $R_{0}(m)$; the corresponding effective value of the magnetic induction of the bending fields, $B_{0}(T)$; the angular frequency $\omega_{R}(\mathrm{~Hz})$ of orbital circulation; and the average total energy lost by an electron during a single revolution about the ring

$E_{1}(k e V)=88.5 \quad E_{0}^{4} R_{0}^{-1}$.

Values of bending radius, magnetic induction, beam energy, and energy loss per revolution because of the emission of synchrotron radiation are conveniently related by the nomograph of Fig. 2 (except when the ring geometry is appreciably noncircular). Additional loss caused by the undulator is, in general, negligible for a storage ring capable of generating photoneutrons in beryllium. Lines on the diagram represent some existing storage rings; the dashed lines represert some planned systems. Note that the highest energy ring shown (PEP) uses weaker bending magnets than some of the other rings. with correspondingly larger ring radius, to constrain the power demand and heat dissipation on the

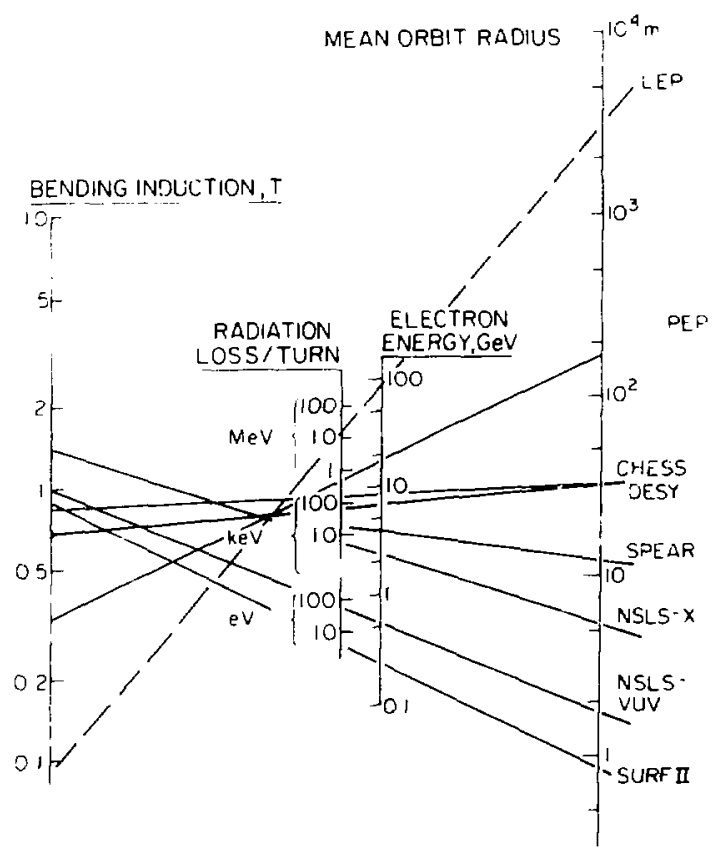

Fig. 2. Relationship of mean orbit radius and bending magnet induction for an approximately circular electron-storage ring, together with the total eneryy loss per revolution from synchrotron radialion. 
walls. Available power is the main obstacle to higher energies; nevertheless, colliding-beam, electron-positron storage rings are being planned for still higher energy. ${ }^{2}$ Beam currents in the range of 0.01 to $1 \mathrm{~A}$ are typical.

Not all the paths of individual electrons in a storage ring lie precisely on a single orbit, nor are they precisely parallel to it; rather, electrons oscillate about the orbit. A measure of their dispersion, termed the "emittance" $i_{i i}$ accelerator parlance, is the area of phase space on a plot of deviation in position vs deviation in direction. This area remains constant as the electrons circulate: at points where an electron is on the orbit, its angular deviation is maximum, while, at its points of miximum excursion from the orbit, it is moving parallel thereto.

Table Il gives some published values of storage-ring emittances.' The finite emittance contributes tc the angular spread of the photon beam and gives the source a finite cross section. The undulator has a relatively minor effect on storage-ring emittance. ${ }^{20}$

\section{NEUTRON PRODUCTION BY MONOENERGEYIC PHOTONS IN BERYLLIUM}

The energy $E_{n} k e V$ of a neutron produced by photodisintegration of ${ }^{9} \mathrm{Be}$ is the excess above binding energy, diminished by recoil. and is given to sufficient accuracy by

$$
\begin{aligned}
E_{n}(\Phi)= & 0.888\left(E_{p}-1665\right) \\
& +1.39 \cos \Phi \mathrm{keV} .
\end{aligned}
$$

where $E_{v}$ is expressed in $\mathrm{keV}$. and $\Phi$ is the angle between the directions of propagation of the neutron and the incident photon."

The fraction of all incident photons that generate neutrons in a target thick in the direction of photon propagation but thin in transverse directions is given by $\sigma_{y, n} / \sigma_{\text {cot }}$. The numerator is the cross section for the photonuclear reaction ${ }^{21}$ and the denominator, that for narrow-beam attenuation. ${ }^{2 ?}$ These cross sections are plotted in Fig. 3. Within a few keV of threshold, the ratio is approximately $2.3(-3)$ neutrons/photon; it decreases to a minimum of approximately $4(-4)$ at $2.2 \mathrm{MeV}$ and rises again to a second maximum of $2.5(-3)$ at $2.9 \mathrm{MeV}$, decreasing at still higher energies. The threshold behavior is not known accurately. Over the energy range we consider here, we can use the constant value $2.3(-3)$.

For simplicity, we assume here that the neutron emission is isotropic. The energy spread of the neutrons is then simply that of the incident photons diminished by the energy-partition factor 0.888 . Estimates of the neutron flux per unit energy interval should include the small spread caused by recoil, given by the second term on the right $|E q .(6)|$ unless the neutrons are observed only in a single direction. In fact, the emission is anisotropic, and the neatrons can be at least partially spin-polarized (Sec. X).

\section{PHGTON PRODUCTION IN AN UN- DULA TOR}

Many detailes derivations of the characteristics of undulator radiation have been published. ${ }^{15.17}$ In thi, section, we summarize relevant relations.

An undulator is characterized as "weak" or "strong." In a weak undulator. electron trajectories are paraxial. transverse velocity is nonrelativistic. and the component of velocity along the beam-axis remains essentially constant. The electric dipole moment oscillates in a purely transverse manner. and only the fundamental frequency is radiated. The radiation in the laboratory system is strongly peaked in the forward direction and fairly monochromatic.

In a strong unjulator, the electron's trajectory is distorted by the magnetic field sufficiently that the transverse component of velocity is also relativistic, the

TABle Il. Typical Storage-Ring Parameters

\begin{tabular}{cccccc} 
Name & $\mathrm{E}_{0}, \mathrm{GeV}$ & \multicolumn{1}{c}{$1, \mathrm{~A}$} & $\mathrm{e}_{\mathrm{x}}, \mathrm{mrad}$ & $\mathrm{e}_{\mathrm{y}}, \mathrm{mrad}$ & Ref. \\
\hline VUV & 0.7 & 1.0 & $9(-8)$ & $9(-10)$ & $3-10$ \\
SSR:L & 3 & 0.2 & $\ldots$ & $\ldots$ & $3-10$ \\
LEP & 86 & 0.009 & $8(-8)$ & $\ldots$ & 2 \\
ESRF & 5 & 0.565 & $1(-8)$ & $1(-9)$ & 26 \\
\hline
\end{tabular}




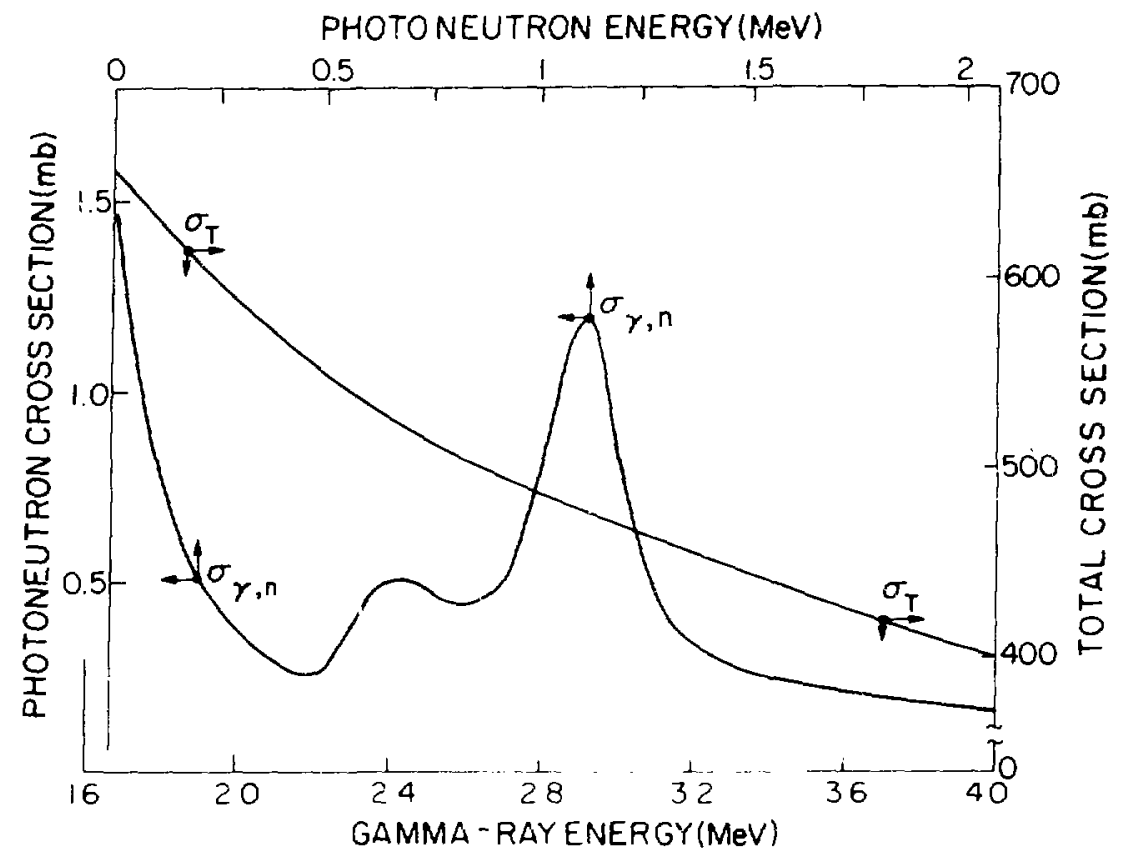

Fig. 3. Comparison of :ross secumons fur ptoton attenuation (right margin) and for photoneutron production (Ieft margin) in beryllsum. Note the l.fos $\mathrm{MeV}$ gamma ray threshold energy, the resonance peaks for the $(\gamma, n)$ reaction and the suppressed zero scale for the total cross section.

total distance traversed within the undulator is slightly increased, and the axial component of velocity varies periodically at twice the fundamental frequency. In the forward direction. only odd harmonics are present, but in the off-axis direction, even harmonics are presert as well because of varying axial momentum.

Undulator strength is expressed by the factor ${ }^{15}$

$$
K=0.934 \lambda_{u} B_{u}
$$

with $\lambda_{u}$ in $\mathrm{cm}$ and the amplitude $B_{v}$ of the magnetic induction given in $T(10 \mathrm{kG})$. Weak undulators have $\mathrm{K}<$ I: strong undulators $K>2$.

The wavelength of the in-th harmonic radiated by electrons of energy $\gamma \mathrm{mc}^{2}$ and observed at an angle $\theta$ to the beam is given by ${ }^{\prime \prime}$

$$
\mathrm{m} \hat{\lambda}_{\mathrm{m}}=\left(\frac{\lambda_{\mathrm{u}}}{2 \gamma^{2}}\right)\left(1+\frac{\mathrm{K}^{2}}{2}+\gamma^{2} \theta^{2}\right)
$$

The spectral distribution of the radiation is discussed in the Appendix. which shows th.' the full bandwiden is

$$
\frac{\Delta i_{m}}{i_{m}}-\frac{\Delta E_{m}}{E_{m}}=(m N)
$$

in the axial direction $(\theta=0)$. Because of the term $\theta$ in Eq. (8), off-axis radiation has slightiy different wavelength, and the line is correspondingly broader when off-axis radiation is accepted (see Appendix). However, on axis, the bandwidth in the first harmonic from a 1000 element undulator is only $0.1 \%$ of the photon energy; if the latter is just above the neutron threshold of ${ }^{9} \mathrm{Be}$, the energy spread of the neutrons is only $1.7 \mathrm{keV}$, comparable to the energy spread caused by :ecoil the second term in Eq. (6).!. However, this spread would be true only "near the axis": that is, within the angle

$\theta_{\text {max }}=\left(\because V^{\prime} N\right)^{-1}$ 
To restrict the radiation to such a small aperture poses a difficult experimental problem. A pinhole aperture for 1.7-MeV gamma radiation is not practicable. However, we shall see in Sec. IX that collimation is unnecessary because the radiation outside $\theta_{\max }$ usually does not contribute to neutron production.

Keeping the electron-beam emittance low enough that the narrow-band, highly collimated photon emission is not spread into a larger solid angle by the dynamics of focussing in the storage ring, by magnet imperfections and misalignments, and by quantized energy losses of electrons in the undulator is a more serious challenge.

The intensity of radiation emitted in the m-th harmonic along the axial direction is found from the axial brightness relation.

$\beta_{m}=4.56(6) N^{2} \gamma^{2} F_{m}(K)$ photons $s^{1} A^{1}$

$(0.1 \mathrm{mrad})^{-2}\left(\frac{\% \Delta E}{E}\right)^{-1}$.

The factor $F_{m}(K)$ is a complicated function (and even more complicated in the off-axis case) expressing the relative intensities of the various harmonics. ${ }^{23}$ The values in Table III have been taken from a graph in Ref. 23.

Within the angle $\theta_{\text {mux }}$. from a weak undulator of $K=$ 0.4 . the number of photons radiated is found /by combining Eqs. (8)-(11)| to be

$J_{1}=6.38(15)$ photons $\mathrm{s}^{-1} \mathrm{~A}^{-1}$.

independent of the number of undulator elements $\mathrm{N}$ and of the electron-beam energy $E_{0}$. which must, of course. be high enough to ensure neutron production within the desired energy range.

\section{ESTIMATE OF NEUTRON YIELD FROM A WEAK UNDULATOR}

Assuming that the $(\gamma, n)$ threshold is indeed exceeded by more than the intrinsic bandwidth of undulator radiation and that the electron-beam emittance is low enough to ensure good collimation, we expect a neutron yield from a weak undulator, $K=0.4$, of approximately

$$
\begin{aligned}
Y_{n} & =6.38(15) \times 2.3(-3) \\
& =1.5(13) \text { neutrons s }{ }^{-1} \mathrm{~A}^{-1}
\end{aligned}
$$

within the angle $\theta_{\max }$. If the undulator generates photons with energy $1.7 \mathrm{MeV}$ and has at least 100 elements, the neutrons then will have average energif $s$ comparable to those from an antimony-beryllium source but much greater energy spread (Sec. IX) and far higher intensity.

\section{UNDULATOR PARAMETERS FOR NEU- TRON GENERATION}

With $\mathrm{K}=0.4 . \mathrm{Eq} .(10)$ yields

$\frac{i_{u}}{y^{2}}=2 \lambda_{1}\left(1+\frac{K^{2}}{2}\right) \leq 1.357(-10) \mathrm{cm}$

if the generated photons are to produce neutrons on beryllium.

From Fig. 2. we see that a $30-\mathrm{GeV}$ electron-storage ring is within present technology; for this ring

$y=5.87(4):$

\begin{tabular}{|c|c|c|c|c|}
\hline \multicolumn{4}{|c|}{ TABLE III. Values of $F_{m}(K)^{n}$} & \multirow[b]{2}{*}{$F_{7}$} \\
\hline K & $F_{1}$ & $\mathrm{~F}_{3}$ & $\mathrm{~F}$, & \\
\hline 0.4 (weak) & 0.14 & $<0.01$ & 0.000 & 0.000 \\
\hline 1.0 & 0.36 & 0.175 & 0.06 & 0.00 \\
\hline 2.4 (strong) & 0.23 & 0.38 & 0.43 & $>0.40$ \\
\hline
\end{tabular}

therefore. 
$\lambda_{\mathrm{u}}=0.468 \mathrm{~cm}$

is the period of the undislator. This period is about one order of magnitude smalier than that of present-day undulators. The corresponding magnetic induction is

$B_{u}=\frac{K}{0.934} \lambda_{u}=0.91 \mathrm{~T}=0.1 \mathrm{kG}$

Although not particularly high. this induction does present some difficulty because so short an undulator period implies a small gap. The undulator therefore would have to be within the vacuum enclosure of the storage ring. That, however, is not a new problem: one design's allows an internal undulator to be removed during the electron-injection operation, then reinserted. or. the beam might be "switched" into the indulator. Precise alignment is essential. however. because. for the 100-element undulator.

$\theta_{\max }=\frac{1}{\gamma \mathrm{N}^{12}}=1.7 \mu \mathrm{rad}$.

At a target $10 \mathrm{~m}$ distant. the radius of the central radiation cone is about $17 \mu \mathrm{m}$ : for full use of the photon beam. the target's length should be at least one absorp tion length for $1.7 \mathrm{MeV}$ photons in beryllium. or

$$
\begin{aligned}
\mathrm{L}_{\text {larget }} & =\geq 11.24(23) \mathrm{cm}{ }^{\prime} \times\left. 660(-27) \mathrm{cm}^{2}\right|^{\prime} \\
& =12 \mathrm{~cm} .
\end{aligned}
$$

Then, the active volume is only $2.7(9) \mathrm{cm}$ '.

The real problems are those of accurate alignment and of maintaining a small electron beam emittance.

The power radiated from the undulator is the product of photon energy times photon generation rate. For a beam current of $0.1 \mathrm{~A}$. the radiated power from the undulator is only $175 \mathrm{~W}$, and only a small fraction of that power is dissipated within the very narrow interac tion volume of the beryllium target because. as already noted. most of the scattered photons and recoil electrons can escape from this volume.

The above figures are time-averages. Because the electrons are bunched in orbit by if cavities, neutron emission is also pulsed. The longitudinal dimension of an electron bunch in the storage ring is determined by the balance of those processes that increase momentum spread and those that tend to restore correct momentum. The former include radiation losses in bending magnets. wigglers and undulators. The $63-\mathrm{mm}$ undulator in Ref. 2 is much shorter than the $46.8-\mathrm{cm}$ undulator discussed above, especially when Lorentz contraction is considered. The duration of each radiation burst, in the above case $1.6 \mathrm{~ns}$, is therefore essentially the transit time of an electron through the undulator. Shorter bursts can be achieved only by shortening the undulator at the expense of increased photon bandwidth. A special technique, recently developed at Frascati." exploits the dependence of path length in the undulator on the electron momentum and can shorten the lengths of electron bunches. but its effect on burst duration is negligible.

The burst-repetition frequency is the product of the number of bunches in the ring by the crbital circulation frequency. Assuming that 10 bunches $2 \mathrm{e}$ in orbit in a ring of $1.2 \mathrm{~km}$ circumference (PEP). the burst repetition frecuuency is $2.4 \mathrm{MHz}$. The duty cycle factor (ratio of active radiating time to total time or the ratio of undulator length to bunch spacing) is

DF $2.4(5) \times 1.6(9) \mathrm{S} \mathrm{Hz}=3.8(-3)$

The peak burst intensity exceeds the average given above by a factor of 260 . so that the peak neutron production rate is $4(15) \mathrm{s}$ '.

The numerical case we have discussed is summarized by Table IV. Other parameters can be considered with the aid of Fig. 4. which shows that. if we are to keep the neutron energy low while using an undulator of longer period $\dot{A}_{u}$. we must increase $E_{o}$. Then. the angular divergence $\theta_{\max }$ is reduced. the burst duration is increased. the total number of neutrons generated remains the same. and the electron-beam emittance requirement is more stringent.

\section{OFF.AXIS BROADENING}

Because we are to work close to the beryllium photorieutron threshold. the angular term in Eq. (8) cannol be neglected when the neutron spectrum is considered. Angular dependence of ihe Doppler effect causes the energy of a photon radiated at an angle $\theta$ to the beam axis to be less than that radiated on the axis by the factor

$$
\frac{E(\theta)}{E(O)}=\frac{1+\left(K^{2} / 2\right)}{1+\left(K^{2} / 2\right)+\gamma^{2} \theta^{2}} .
$$

"Laboratori Nazionali di Frascati dell'INFN, Frascati. Italy. 


\begin{tabular}{|c|c|c|}
\hline \multicolumn{3}{|l|}{$\begin{array}{c}\text { TABLE IV. Possible } \\
\text { Source }\end{array}$} \\
\hline Siorage Ring & & Parameter \\
\hline Electron-beam energy & $\mathrm{E}_{\mathrm{o}}$ & $30 \mathrm{GeV}$ \\
\hline Ring circumference & $2 \pi R_{0}$ & $1.25 \mathrm{~km}$ \\
\hline$\gamma$ & & 58710 \\
\hline Bunch length & & $<10 \mathrm{~cm}$ \\
\hline Horizontal emittance & & (see text.) \\
\hline Energy loss/revolution & & $10 \mathrm{MeV}$ \\
\hline Beam current & & $0.1 \mathrm{~A}$ \\
\hline Number of bunches & & 10 \\
\hline Duty cycle & & $3.8(-3)$ \\
\hline \multicolumn{3}{|l|}{ Undulator } \\
\hline $\mathbf{N}$ & & 100 \\
\hline$\lambda_{2}$ & & $0.468 \mathrm{~cm}$ \\
\hline Total lengeh & & $46.8 \mathrm{~cm}$ \\
\hline Magnetic induction & & $0.91 \mathrm{~T}$ \\
\hline Photon energy & & $1.692 \pm 0.017 \mathrm{MeV}$ \\
\hline Maximum angle for unt & roadened line & $1.7(-6)$ radians \\
\hline Paraxial photon intensit & & $6.5(14)$ photons $s^{\prime}$ within $\theta_{\max }$ \\
\hline Burst duration & & $1.6 \mathrm{~ns}$ \\
\hline Burst-repetition rate & & $2.4 \mathrm{MHz}$ \\
\hline \multicolumn{3}{|l|}{ Neutron Source } \\
\hline Dimensions & & $12 \mathrm{~cm} \times 0.017-\mathrm{mm}$ diam \\
\hline Volume & & $2.7(-3) \mathrm{cm}^{3}$ \\
\hline Neutron energy & & $0-30 \mathrm{keV}$ \\
\hline Neutron yield (time ave & aged) & $1.5(12) \mathrm{ns}^{1}$ \\
\hline Neutron yield (pulsed) & & $4(14) n s$ \\
\hline Target heat rate & & $175 \mathrm{~W}$ \\
\hline
\end{tabular}

When $\mathrm{E}(\mathrm{O})$ is close to threshold, the angular factor rapidly cuts of neutron production by off-axis photons (Figs. 5 and 6). The $1 \%$ bandwidth lines for each case are displayed in the figures.

Without undertaking a more elaborate analysis, we therefore can assume that all photoneutron generation effectively occurs within the angle $\theta_{\max }$ given by Eq. (10) when we are working close to threshold. The neutron yield estimated in Sec. VIII is correct in order of magnitude, and collimation is not necessary.
The angular dependence smears out the neutron spectrum. Figure 6 shows that, even though on-axis photons within $0.5 \%$ of $1690 \mathrm{keV}$ generate neuts uns of energy in the range $15-29 \mathrm{kEV}$, the off-axis photons extend the spectrum practically to zero. With lower central energy (Fig. 3 , some of the photons within the bandwidth are below the $(\gamma, n)$ threshold.

Figure 7 shows neutron spectra calculated by the analysis outlined in the Appendix for two values of the central photon energy: 1.68 and $1.69 \mathrm{MeV}$, respectively. 


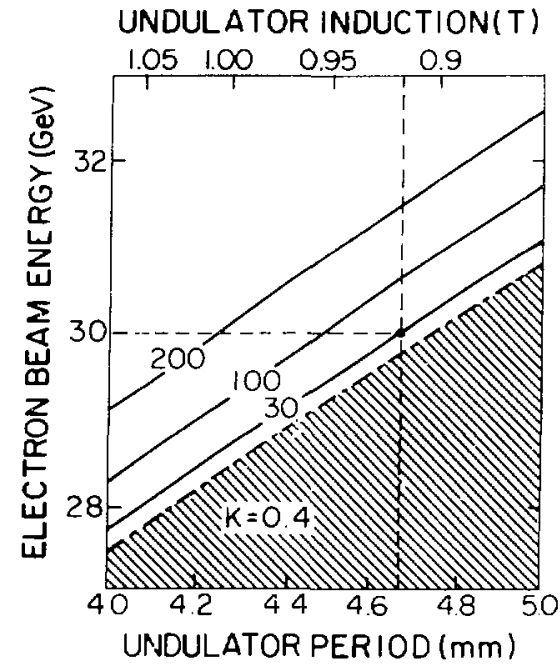

(a)

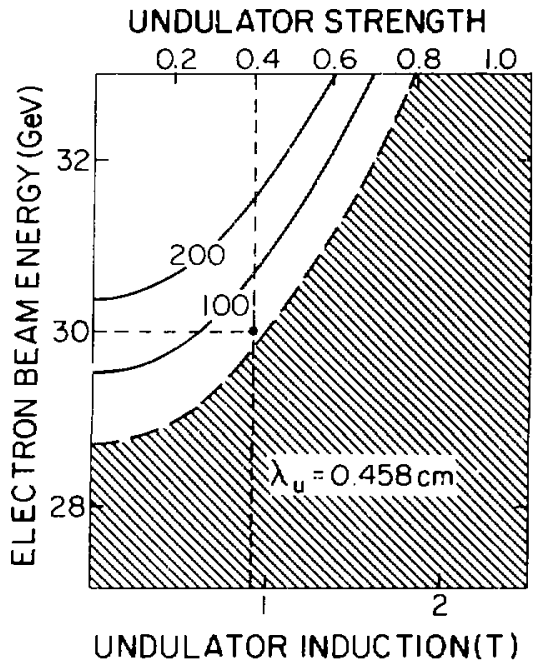

(b)

Fig. 4. Effect of varying undulator ?:riod, induction, or electron-beam energy on the average ener'y of the photoneutrons from heryllium (parameter for the curves. given in keV). The values taken for illustration in the text are indicated by the solid point.

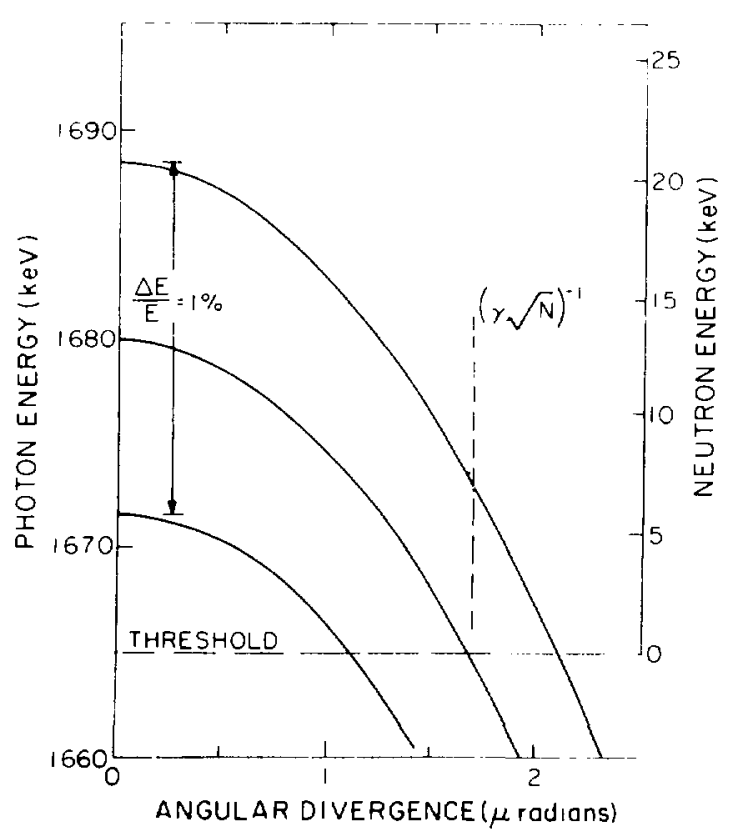

Fig. 5. Angular dependence of the energy of generated photons, showing the energy of maximum intensity and the energy at the first zero on either side of the inaximum, for a 100 -element undulator in a $30 . \mathrm{GeV}$ storage ring. $\mathrm{W}: \mathrm{h}$ the maximum adjusted to $1.68 \mathrm{MeV}$, the average neutron energy is $13.5 \mathrm{keV}$.

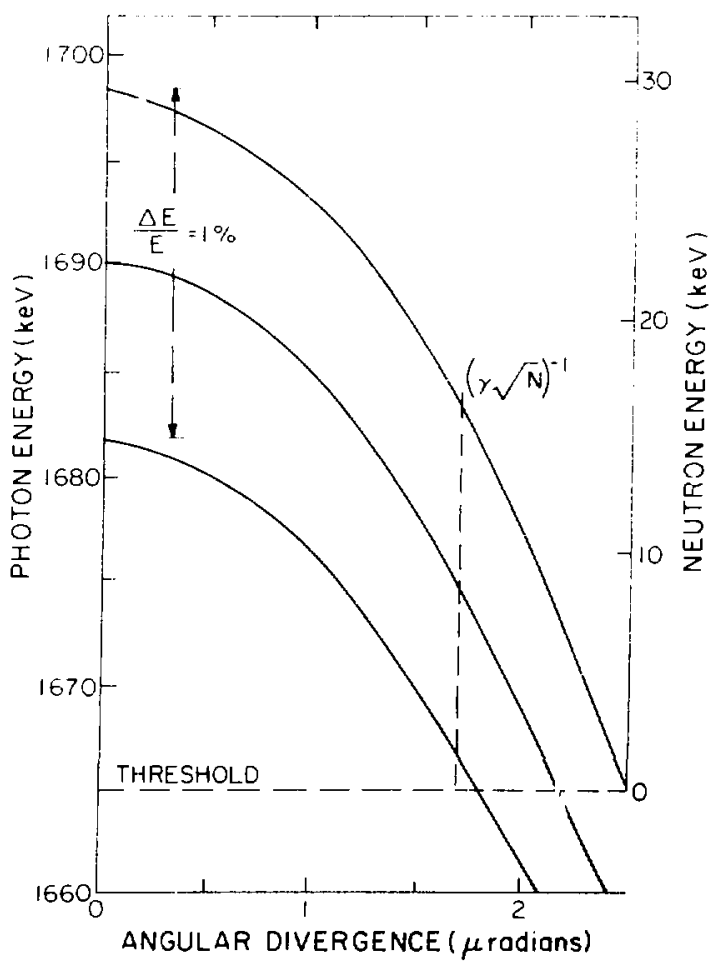

Fig. 6. The same as Fig. 5, except that the energy of maximum intensity has been increased to $1.69 \mathrm{MeV}$, shifting the average energy of the neutron spectrum to $22.5 \mathrm{keV}$. 


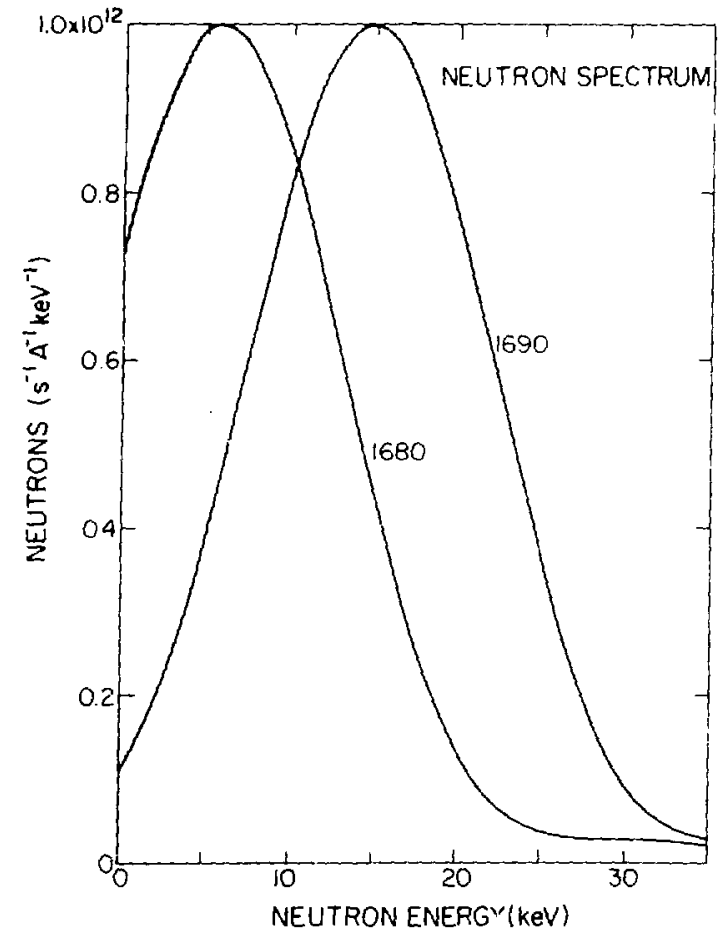

Fig. 7. Spectral distribution of the neutron intensity for two different values of the central energy of the gamma-ray line. The intensity is given in neutrons pet ampere of circulating beam current per second, in a $1-\mathrm{keV}$ energy bandwidth. The undulator contains 100 elements, giving a gamma-ray bandwidth of $1 \%$.

The abrupt jump to a finite vaiue at zero neutron energy is probably spurious because it assumes similar behavior in the $(\gamma, n)$ cross section of beryllium More accurate data on this cross section near threshold are necessary to determine this part of the spectrum; however, the qualitative behavior at higher neutron energies is probably correct. A region of interest in the neutron spectrum can be selerte'd by making only slight changes in the undulator or electron-beam parameters. The slight tail on the high-energy side is a consequence of secondary maxima in the photon spectrum; these damp out rapidly, however.

We have not considered finite divergence of the electron beam in the undulator, nor, if its divergence is zero, its finite radial extent. Each has the effect of further smearing out the neutron spectrum seen at any radius within the target not too far off-axis. To avoid "emit- tance smearing" of the spectrum, the electron-beam emittance must be less than $3(-11)$ mrad.

\section{POLARIZATION}

Circularly polarized photons can be obtained by mieans of a helical undulator; or plane polarized phorons can be obtained by using a planar undulator that can be sotated about the beam-axis to control the plane of polarization. These facts are importart in considering the value of an undulator neutron source.

Near the photoneutron threshcld of beryllium, the reaction is dominated by the $(1 / 2-$ level, located at approximately $1.67 \mathrm{MeV}^{24}$ above the ground state $(3 / 2+)$ and therefore involves an electric dipole transition. The neutron carries of a' the free angular meinentum because the ground state of ${ }^{\mathrm{B}} \mathrm{Be}$ has zero spin.

With these parameters, the neutrons from an unpolarized beryllium target irradiated with circularly polarized photons will be emitted isotropically with a degree of polarization proportional to the sine of the angle between the directions of propagation of the photon and the neutron." More complicated dispen. dences are expected with a planar undulator or with a polarized beryllium target.

\section{NEUTRON MODERATION}

The $1.5(13)$ neutrons per ampere intially generated each second $\{6.3(6)$ per burst $\}$ within the filamentary region $12 \mathrm{~cm}$ long and $17 \mu \mathrm{m}$ in radius** immediately emerge from within that small volume $\left|2.7(-9) \mathrm{cm}^{3}\right|$ and make their first collisions elsewhere. If the neutron spectrum does not require moderation for the interided application, then the source is extremely compast both spatially and temporally; however, if more neutrons of lower energy are required, then the target must be immersed in a moderator with substantial increase in effective source size and burst duration. An additional volume of beryllium outside the beam-axis or a water bath can be used as a moderator to delay and spreao out

"Prof. Kenneth Krane, Dept. of Physics, Oregon State University, Corvallis, Oregon, provided th., information.

*When the target is at the assumed 10-m distance; for other distances, the radius is proportionately changed, but in the absence of beam attenuation, the neutron yield is unchanged. 
the low-energy part of the neutron spectrum over an interval much longer than the iritial burst duration.

The average time $t_{m}$ required for a neutron to moderate from an initial energy $E_{1}$ (eV) to a lower energy $E_{2}$ (eV) by elastic collisions in water is given very nearly by the relation ${ }^{24}$

$t_{m}=0.8(-6)\left\{\left(E_{2}\right)^{-1 / 2}-\left(E_{1}\right)^{-1 / 2}\right\} s$

in Fig. 8. The times in this sure are for a neutron to moderate from an infinite energy. If, lor example. a neutron with an initial energy of $10 \mathrm{keV}$ is moderated to $1 \mathrm{eV}$, the corresponding times, $0.8 \mu \mathrm{s}$ and $8 \mathrm{~ns}$. respectively, are subtracted. Statistical fluctuations of the moderation time spread out the moderated burst into an interval comparable with the moderation time itself, in the case of water.

If, instead of water, one moderate: the neutrons in beryllium, then the moderation times sho..ld be increased approximately sevenfold to take account of the longer mean free path $(1.6$ vs $11 \mathrm{~cm})$ and lower lethargy increase per collision $\left(0.209\right.$ vs 1.00). ${ }^{24}$ The time-spreading will be a smaller fraction of the total

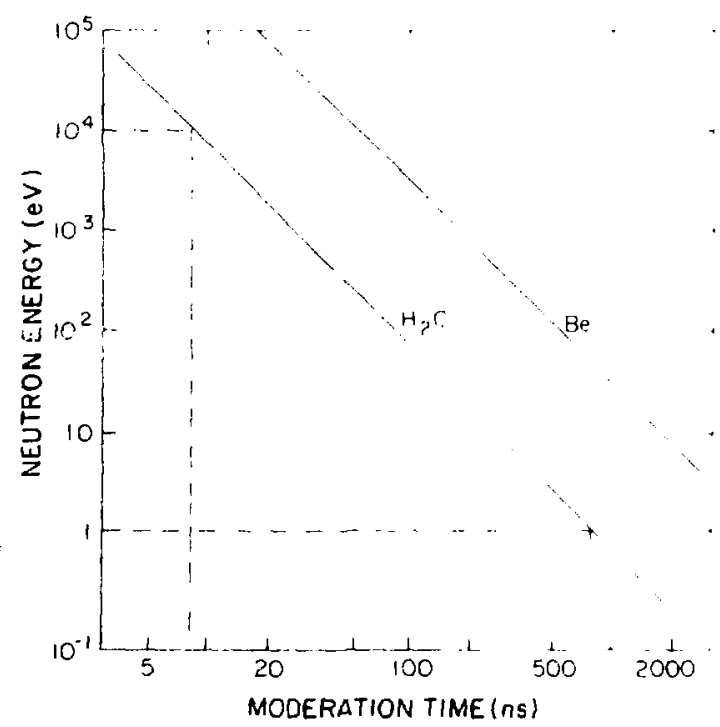

Fig. 8. Times for neutrons to moderate from essinually infinite initial energy to the energy shown as ordinate. for water, and for beryllium of normal density. For finite intial energy, diflerences should be taken; for example, moderation in water from $10 \mathrm{keV}$ lo $\mathrm{I} \mathrm{CV}$ requires $750-9=741 \mathrm{~ns}$. on the average. (Statistical Muctuations in these cimes are com parable with the total moderation time in the cas: of water.) moderation time. but its absolute value will be larger than that of water.

The spatial extent of the moderator is also comparable to that needed to moderate neutrons from a source of much higher energy. The slowing down distances in the two cases are approximately in the ratio of the square roots of the corresponding lethargy ${ }^{25}$ increases. For exampie, to moderate fission neutrons from $2 \mathrm{MeV}$ to 1 eV (lethargy increase of 6.3 ) requires a moderator radius only 1.2 times idrger than 1, moder ate from $20 \mathrm{keV}$ to I $\mathrm{eV}$ (lethargy increase of 4.3 ).

Therefore, even though the original generation process is very brief, an undulator photoneutron source (UPS) with a moderator gives only a slight advantage, if any, over other sources with respect to neutron-burst duration. unless the values of $K$ or $E_{0}$ are adjusted so that only very low energy neutrons are produced. because most of the time for moderation is spent at low energy

Therefore. the undulator photoneutron source may not find application as a source of thermat or near thermal neutrons unless special applications dictate the absence of nig,i-energy neutrons (for example. where material damage. however slight. must be prevented) or unless a moderator that operates by inelastic neutron scattering is employed. However. we have not in vestigated that possibility. The main rationale for de veloping this source must come from applications that use resonance-energy and polarized neutrons and that require short hurst duration without moderation.

\section{THE STRONG UNDULATOR CASE}

$A$ weak undulator such as that described in Sec. XI. with $K=0.4$, radiates only at the fundamental undulator frequency | sec Table III and Eq. (13)]. Because strong undulators also generate harmonics with line widths inversely proportional to the order $m$ of the harmonic and values of $F_{m}(K)$ higher than $F_{t}(K)$, their use seems worthwhile. The following argument shows that this is not the case for a photoneutron source.

If we wish to generate $1.69 \cdot \mathrm{MeV}$ photons, the value of

$$
\left(\frac{i_{u}}{2 m \gamma^{2}}\right)\left(1+\frac{K^{2}}{2}\right)=7.34(-11) \mathrm{cm} .
$$

If we take a strong undulator with $K \sim 2.4$, such that the fifth harmonic $m=5$ is the most intense and adjust $K$ to make its energy $E_{9} \approx 1.69 \mathrm{MeV}$, we have a new value for 
$\lambda_{u} / \gamma^{2}$, which might be realized by keeping the former value of either $\lambda_{u}$ or $\gamma$, or by readjusting both, subject to constancy of their combination. Table $V$ shows the results of the former.

We conclude that the strong unduiator offers only small advantages of undulator spacing, beam energy. and intensity at the expense of requiring far higher magnetic fields. Furthermore. the lower harmonics that can not generate neutrons nevertheless contribute to target heating. while the still higher harmonics also present in appreciable numbers generate neutrons of much higher energy.

\section{OTHER POSSIBILITIES}

Hofman ${ }^{2}$ has discussed two other possibilities for neutron generators having a storage ring as the primary element in the planned $86 \mathrm{GeV}$ LEP ring at CERN. One is to use wiggier radiation ci energy and bandwidth high enough to cover the giant photonuclear rescnance to produce a broad spectrum of neutrons with a max. imum energy near $1 \mathrm{MeV}$ and a total intensity of the order of $2.5(14) \mathrm{s}^{-1} \mathrm{~A}^{-1}$. However, the target dissipation would be over $100 \mathrm{~kW}$. The other is to use Compton backscattering of optical laser photons. Although the latter can generate very high photon energies for nuclear research. ${ }^{26.27}$ it is not well-matched to neutron-generation requis ements, and intensities are limited. A microwave cavity could be used in place of the undulator to set up a pattern of standing waves so that photons of very low frequency are scattered from fast electrons. This un dulator would be very weak. however: $F,(K)$ would be

"European organization for nuclear re rch. laboratory at Geneva. Swizerland. very small at any reasonable microwave power level. We have not attempted a quantitative assessment of these alterhatives. For more information, consult Ref. 2.

\section{APPLICATIONS}

Eremeev's proposa!' suggests that the intensity. spec trum and geometry of a synchrotron-radiation photoneutron source is ideal for TOF nestron spectrometry, for experiments on neutron-neutron scattering. and for pumping a graser.

\section{A. Time of Flight Neutron Spectrometry}

We consider a neutron TOF spectrometer in Fig. 9. A 100 element undulator in a $30 . \mathrm{GeV}$. 1-A storage ring generates a beam of polarized bamma radiation of energy slightly above the photoneutron threshold of theryllium. with approximately $17-\mathrm{keV}$ energy spread. Figure 7 shows that the total photoneutron spectrum. centered at approximately $8 / 9$ of the excess photon energy above the $1.665 \mathrm{keV}$ threshold. has a full width at half intensity of about $16.5 \mathrm{keV}$ : a "region of interest" centered at $15 \mathrm{keV}$ extends from nearly 0 to above 25 $k e V$ without difficulties from decreased intensity. The central energy of the ncutrun spectrum can be shifted up or down by (1) slight changes in the magnetic field of the undulator. (2) changing the energy of the electrons in the storage ring. or (3) slight lateral displacement of a filamentary beryllium target.

The spectral shift of off axis photons. discussed in Sec. IX. makes the complete absence of very slow neutrons unlikely. Without better data on the near-threshold behavior of tine $(Y, n)$ cross section, we cannot give an

TARLE V. Comparison of Weak and Strong Undulators Generating I.6́9 MeV

\begin{tabular}{|c|c|c|c|c|c|c|}
\hline $\mathbf{K}$ & $\mathbf{m}$ & $3 \sqrt{ }, 2, \mathrm{~cm}$ & $i_{u}, \mathrm{~cm}$ & $\gamma$ & $B_{u}, T$ & $\mathrm{E}_{\mathrm{o}}, \mathrm{GeV}$ \\
\hline Weak 0.4 & 1 & $1.359(\cdots 10)$ & 0.468 & $5.87(4)$ & 0.91 & 30 \\
\hline Strong 2.4 & 5 & $1.892(-10)$ & $\begin{array}{l}0.652 \\
0.468\end{array}$ & $\begin{array}{l}5.87(4) \\
4.97(4)\end{array}$ & $\begin{array}{l}3.94 \\
5.49\end{array}$ & $\begin{array}{c}30 \\
25.4\end{array}$ \\
\hline
\end{tabular}




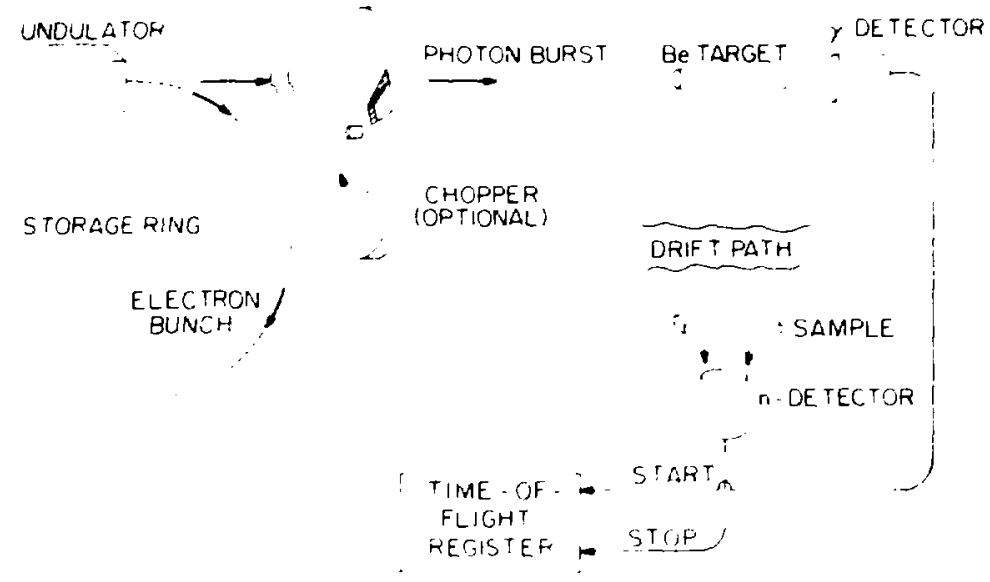

Fig. 9. A neutron spectrometer using a 1'PS at a pulsed neutron source The chopper is an iptional feature.

accurate description of the spectrum at near-ztro energy. If the cross section does rise gradually rather than abruptly as the gamma ray energy is increased above threshold. frame overlap in TOF work with this type of pulsed source may not be a problem. However. if frame overlap does become troublesome, the beam of undulator radiation can be chopped with a mechanical fasi chopper (Fig. 9). having a rotation period equal to the desired burst spacing and an aperture open just for one normal period between lindulator radiation bursts (the orbital circulation period of the storage ring. in single bunch mode). To design a neutron beam chopper - with an open interval of only $400 \mathrm{~ns}$ is a formidable task. On the other hand, because the gamma-ray beam is only

- a few micrometers in diameter, it can be e.sily chopped.

- An aperture of suitable size could be mate by laser machining so a velocity of about $1 \mu \mathrm{m} / \mathrm{ns}$ is quite practicable. We might vibrate the beryllium target by a piezoeiectric driver with amplitude sufficient to take it outside the central part of the gamma-ray beam. Alternatively, the eleciron beam could be switched into the undulator snly at selected times.

Perhaps the best method is to modulate or pulse the undulatc: magnets. A change of $0.25 \mathrm{~T}$ in the undulator magnetic field can change the gamma-ray energy by 75 $\mathrm{keV}$, enough to modulate from below-threshold to well above it.

The neutron source will be long. about $12 \mathrm{~cm}$. as shown above, unless we accept reduced neutron yield. bue the radial dimension of the neutron source can be extremely small because of the high collimation of undulator radiation and becausc no moderator is re quired. For minimum uncertainty in flight path. the neutrons emitted normally to the axis of the undulator would be observed. This restriction introduces some disadvantages in sample dimensions.

Auchampacgh ${ }^{28}$ has presented a comparison of sever al accelera'or based neutron spectrometry facilities. Fig. ure 10. taken froni his paper, shows the result of applying his method to the UPS adjusted to $1.69 \mathrm{MeV}$. The undulator pulsed source produces a relatively nar row but intense spectrum of neutrons in the region between 1 and $100 \mathrm{keV}$. This source can be shifted in energy with litule change in shape by varying undulator or storage ring parameters. Its advantages are (1) the neutron polarization can be adjusted. including essentialIy complete polarization: (2) no moderator is required: and (3) the frame-overlap problem can be eliminated without gating the source.

In Auchampaugh's comparison. the timing uncertain ty is taken to be $1.6 \mathrm{~ns}$. The neutrons are assumed to be detected normaliy to the :xis of the $12-\mathrm{cm} \cdot$ long source filament. To achieve an energ' resolution of $8.8(-4)$, the flight disiance is $79 \mathrm{~m}$. Using the neutron spectrum of Fig. 7 to calculate neutron flux at the detector position yneids the dashed curve in Fig. 10. The UPS compares favorably with any of the other facilities. particularly when we consider the advartages listed above.

Table $V I$ presents estimates of time of light (TOF). neutron intensity per burst, and energy resolution $\delta \mathrm{E}$ for two much shorter values of light path and for a range of 


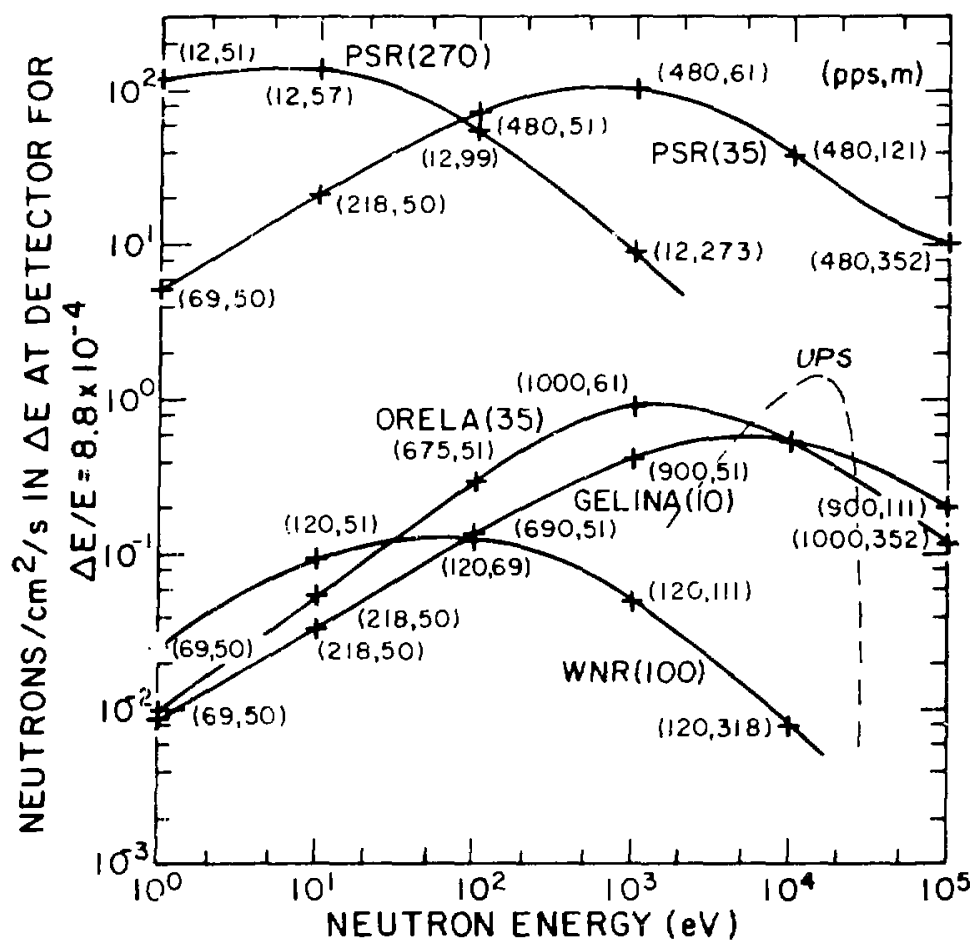

Fig. 10. Comparison of a number of facilities for neutron spectrometry. all adjusted for a common energy sesolution of $8.8($ 4). (Adapted from Ref. 28. )

TABLE VI. Neutron TOF Parameters

\begin{tabular}{|c|c|c|c|c|c|c|}
\hline $\begin{array}{l}\text { Neutron Energy } \\
\text { Neutron Velocity }\end{array}$ & & $\begin{array}{c}0.1 \\
1.39(7)\end{array}$ & $\begin{array}{c}1.0 \\
4.38(7)\end{array}$ & $\begin{array}{c}10 \\
1.39(8)\end{array}$ & $\begin{array}{c}100 \\
4.38(8)\end{array}$ & $\begin{array}{l}\mathrm{keV} \\
\mathrm{cm} / \mathrm{s}\end{array}$ \\
\hline $\begin{array}{l}\text { Flight path I m } \\
4 \mathrm{n} / \mathrm{cm}^{2} / \text { burst } \\
\delta \mathrm{L} / \mathrm{L}=0.18 \%\end{array}$ & $\begin{array}{l}\text { TCF } \\
\delta \mathrm{TOF} / \mathrm{TOF} \\
\delta \mathrm{E}\end{array}$ & $\begin{array}{l}7.22 \\
0.02 \\
0.18\end{array}$ & $\begin{array}{l}2.38 \\
0.07 \\
1.92 \\
\end{array}$ & $\begin{array}{l}0.72 \\
0.22 \\
28.6\end{array}$ & $\begin{array}{l}0.238 \\
0.88 \\
695 \\
\end{array}$ & $\begin{array}{l}\mu s \\
\% \\
\mathrm{eV}\end{array}$ \\
\hline $\begin{array}{l}\text { Flight path } 10 \mathrm{~m} \\
4 \mathrm{n} / \mathrm{cm}^{2} / 100 \text { bursts } \\
\delta \mathrm{L} / \mathrm{L}=2(-3) \%\end{array}$ & $\begin{array}{l}\text { TOF. } \\
\delta \text { TOF/TOF } \\
\delta E\end{array}$ & $\begin{array}{l}72.2 \\
0.002 \\
0.003\end{array}$ & $\begin{array}{l}23.8 \\
0.007 \\
0.070\end{array}$ & $\begin{array}{l}7.22 \\
0.022 \\
2.2\end{array}$ & $\begin{array}{l}2.38 \\
0.067 \\
67\end{array}$ & $\begin{array}{l}\mu s \\
\% \\
e V\end{array}$ \\
\hline
\end{tabular}

neutron energies. The estimated energy resolution includes neither detector time-jitter nor finite effective detector thickness and therefore may be unduly optimistic.

\section{B. Neutron-Neutron Scattering}

By placing two filamentary beryllium sources side by side, using two slightly displaced undulators for generating the exciting gamma-radiation or a single strong 
undulator with two beryllium filaments, each displaced from the central axis, we can hope to observe a neutron-neutron interaction.'

Unfortunately, the energy distribution of the photoneutrons, even with a many-element undulator, is still so broad that detecting an effect uniquely ascribable to neutron-neutron scattering may be difficult. Ignoring this practical question, we simply estimate an upper bound to the number of neutron-neutron scattering events that may occur per unit time, taking an average cross section of 5 b to obtain an order-of-magnitude estimate.

If the two sources each emit $S$ neutrons in the form of $F$ bursts of duration $T$ each second, and if $V$ is the total volume occupied by these neutrons at the end of the burst in a cylinder of radius $\langle v\rangle$ and length $L+2\langle v\rangle T$ $\sim \mathrm{L}$ (where $\mathrm{L}$ is the length of the source in the direction of the radiation-axis), then the density of neutrons contributed by either of the sources is

$\mathrm{n}=\frac{\mathrm{s}}{\mathrm{FV}}$.

and the number of scattering events in the neutron-occupied volume is about

$R=n^{2}\langle v\rangle \sigma V T F$.

Combining. we obtain for the interaction rate

$$
R=\frac{S^{2} \sigma}{\pi<v>L T F .}
$$

Taking the values

$$
\begin{aligned}
& \mathrm{S}=1.5(13) \mathrm{n} \mathrm{s}^{-1}, \\
& \sigma=5(-24) \mathrm{cm}^{2}, \\
& \mathrm{v}=1.8(9) \mathrm{cm} \mathrm{s}^{-3}, \\
& T=1.6(-9) \mathrm{s}, \text { and } \\
& \mathrm{F}=2.5(6) \mathrm{Hz},
\end{aligned}
$$

we obtain only $1.6(-5)$ events per second, barely one per day! We conclude that this approach is not feasible for any reasonable storage-ring current.

\section{Gamma-Ray Laser Pumping}

The possibility of pumping a graser in the form of a doped filament of beryllium by exposing it to an intense burst of neutrons has been considered many times, ${ }^{12,13,24,30}$ and the idea has undergone many variations. ${ }^{29}$ Estimated neutron densities required to achieve a population inversion are too high for realization by moderation of neutrons from even an explosive fission source. ${ }^{31}$ Recently, Karyagin ${ }^{30}$ has called attention to the Eremeev proposal. ${ }^{1}$ Neuirons from a photoneutron source require less moderation and therefore impart less heat to a moderator. ${ }^{31}$ The geometry of the photoneutron source we consider here is identical to that of the proposed graser. where the photoneutron reaction would be initiated sequentially down its length, as in a trav. eling-wave pump. to aid in selecting a sense of propagation for the developing wave of stimulated emission from the neutron-generated isomers. The duration of the neutron burst would be much shorter than that of moderated fission neutrons, and, therefore, better matched to the rather short lifetimes of the Mössbauer transitions essential to any conceivable graser device.

On the other hand, the neutron yields still may not be high enough. A number of recent suggestion ${ }^{29,30}$ have been made that, taken in combination. might lower the excitation requirements for lasing so that the undulator photone.ttron source might inceed make a suitable pump.

\section{COMMENTS}

Altnough the neutron yields calculated in Sec. VII and $X I V$ are not as high as those of fission and spallation sources and are lower than Eremeev ${ }^{1}$ estimated, they are high enough for interesting applications to exploit the short pulse length. low-energy spread, controllable polarization. and line geometry unique to this type of source. We therefore urge further study of this possibility.

The practicality of this concept depends on whether the accelerator engineer can meet the challenges of low beam emittance and precise alignment: design parame. ters more severe than for other applications. Most storage-ring facilities are built either for high-energy physics research or for production of lower energy photons. Therefore, they are available only for occasional parasitic operations if, indeed, they can be used for neutron generation at all. An entire system designed for and dedicated to neutron production is necessary for full realization of the potential of this concept.

Although the parameters of a storage ring for neutron production must be closely specified and kept within 
narrow limits, many other important uses for the complete storage-ring system need not be precluded. The synchrotron radiation from other parts of the ring can be useful for any research that benefits from high critical energy (30l keV), short critical wavelength $(0.4 \mathrm{~nm})$, excellent collimation, and extreme stability. Besides the wide variety of current applications at existing facilities, medical (both therapeutic and diagnostic) and nuclear (excitation of low-lying levels with polarized photons, instrument development, and calibration) applications should be possible with this unique system.

\section{ACKNOWLEDGMENTS}

I thank A. Sandorfi, A. Van Steenbergen, R. Chrien, and $A$. Luccio of Brookhaven National Laboratory for helpful discussions during a recent visit to BNL's National Synchrotron Light Source facility; A. Bienenstock and $H$. Winick of the Stanford Synchrotron Radiation Laboratory for encouraging suggestions and information provided during a recent visit to their facility: George Auchampaugh, John Browne, Michael Moore, and John Moses for frequent consultation; and Professor Kenneth Krane of Oregon State University. Corvallis, Oregon for a discussion of polarized neutron production.

\section{APPENDIX}

\section{SPECTRAL DISTRIBUTIONS}

Undulator-generated photons have the energy distribution $^{2}$

$$
\frac{d^{2} I}{d \Omega d E}=\frac{C N \gamma^{2}}{E(\Theta)} f_{N}\left(\frac{\Delta E}{E}\right) F_{u}(\theta, \phi) .
$$

where $\mathrm{C}$ is a constant,

$f_{N}(x)=\frac{\sin ^{2} N \pi x}{(N \pi x)^{2}}$,

and

$$
F_{u}(\theta, \phi)=\frac{1+2 \gamma^{2} \theta^{2}\left(1-2 \cos ^{2} \phi\right)+\gamma^{2} \theta^{2}}{\left(1+\gamma^{2} \theta^{2}\right)^{3}},
$$

where the value of $\gamma$ is that of average axial velocity in the undulator, rather than that in the storage ring.

The solid angle element is well approximated by

$$
d \Omega=\theta d \theta d \phi
$$

so that, after integrating over the axinuthal angle $\phi$, we obtain

$$
\begin{aligned}
& \frac{\mathrm{dI}}{\mathrm{dE}}=2 \pi \mathrm{CN} \gamma^{2} \\
& \int_{0}^{\mathrm{H}_{-} / \mathrm{N}} \frac{1}{\mathrm{E}(\theta)} \frac{\sin ^{2}(\mathrm{~N} \pi \Delta \mathrm{E} / \mathrm{E})}{(\mathrm{N} \pi \Delta \mathrm{E} / \mathrm{E})^{2}} \theta \mathrm{d} \theta .
\end{aligned}
$$

The energy deviation $\Delta \mathrm{E}$ is a function of the angle $\theta$. in accordance with

$$
\mathrm{E}(\theta) \doteq \mathrm{E}(0)\left(1+\gamma^{2} \theta^{2}\right)^{-1} .
$$

Evaluation requires expansion of the $\sin ^{2} x / x^{2}$ function. inserting Eq. (A-6), and integrating term by term. The resulting series converges rapidly after the first iew terms and can be evaluated on a programmable desk calculator. In contrast to other analyses (for example, Ref. 23) we do not extend the limit of integration to large values o! $\theta$ where there is no contribution to photoneutron generation.

Converting photon energy to neutron energy by subtracting the threshold and applying the factor $8 / 9$ yields Fig. 7.

Note that Eq. (A-2) gives zero intensity at two points separated by the interval $E / N$. This is the basis for $E q$. (9), the expression of photon bandwidth. Secondary 
maxima on either side. whose contribution to the spec trum at higher energies accoun's for the weak tail visible on the $1680 \mathrm{keV}$ curve of Fig. 7. rapidly damp out.

\section{REFERENCES}

1. P. Eremeev, "Neutron Focus in Beryllium in a Synchrotron Radiation Field," Sov. Phys. JETP Lett. 27. (10 January 1978).

2. A. Hofman. "Synchrotron Radiation from the Large Electron-Positron Storage Ring LEP," Phys. Rep. 64. 253 (September 1980).

3. E. M. Rowe. "Synchrotron Radiation Facilities in the United States." Phys. Toda, 34. 28 (May 1981).

4. C. J. Sparks. Jr.. "Synchrotron Radiation with X Rays." Phys. Today 34, 40 (May 1981).

5. H. Winick. G. Biuwn. K. Halbach. and J. Harris, "Synchrotron Radiation Wiggler and Undulator Magnets." Phys. Today 34, 50 (May 1981).

6. D. E. Eastman and F. J. Himpsel. "Synchrotron Radiation-an Incisive and Versatile Tool," Fhys. Today 34. 64 (May 198I).

7. Proc. of the National Conference on Synchrotron Radiation Instrumentation, K. Siegbahn-Uppsala, Ed.. Nucl. Instrum. Methods 172. No. 1 (May 1980).

8. K. R. Lea. "Highlights of Synchrotron Radiation," Phys. Rep. 43, 339 (July 1978).

9. G. N. Kulipanov and A. N. Skrinskii, "Utilization of Synchrotron Radiation: Current Status and Prospects," Sov. Phys. Usp. 20. 559 (Juiy 1977).

10. Synchrosron Radiation Research. H. Winick and S. Doniach, Eds. (Plenum Press, New York, 1979).

11. B. T. Feld, "The Neutron," in Experimental Nuclear Physics, E. Segre. Ed. (John Wiley and Sons, New York, 1953), Part VII, Sec. 3, 357-460.
12. V. I. Gol'danskii and Yu. Kagan, "The Possibility of Creating a Nuclear $\gamma$ Laser," Sov. Phys. JETP 37. 49 (July 1973).

13. V. I. Gol'danskii. Yu. Kagan, and V. A. Namiot. " iwo-Stage Excitation of Nuclei to Obtain Stimu. lated Gamma-Quantum Emission," Sov. Phys. JETP Lett. 18. 34 (July 1973).

14. J. Schwinger. "On the Classical Radiation of AC. celerated Electrons." Phys. Rev. 75. 1912 (May 1949).

15. Y. Farge. "Emission of Photons by Unduluiors." Appl. Optics 19,4021 (November 1980).

16. L. Gevorgy an and N. A. Korkhnazyan. "Undulat i \& Radiation in Dispersive Madia." Sov. Phys. JETP 49. 622 (April 1979).

17. D. F. Alferov: Yu. A. Bashmakov, and E. G. Bessanov, "Undulator Radiation," Sov. Phys. Tech. Phys. 18. 1336 (April 1974).

18. B. M. Kincaid. "A Short-Period Helical Wiggler as an Improved Sourci of Synchrotron Radiation." J. Appl. Phys. 48. 2684 (July 1977).

19. L. R. Elias, W. M. Fairchild. J. M. J. Madey, H. A. Schwettan. and T. I. Smith. "Observations of Stimulated Emission of Radiation by Relativistic Electrons in a Spatially Periodic Transverse Magnetic Field." Phys. Rev. Lett. 36. 717 (March 1976).

20. V. P. Suller. "The Interaction of Wigglers and Undulators with Stored Electron Beams." Nucl. Instrum. Methods 172.39 (May 1980).

21. B. L. Berman, R. L. Van Hemert. and C. D. Bowman. "Threshold Photoneutron Cross Section for Be"," Phys. Rev. 163. 958 (Novemher 1967).

22. E. storm and H. Israel. "Photon Cross Sections $0.00 \mathrm{i}-100 \mathrm{MeV}$ for Elements 1 through 100," Los Alamos Sciertific Laboratory report LA-3753 (June 1967). 
23. S. Krinsky, "An Undulator for the $700 \mathrm{MeV}$ VUV Ring of the National Synchrotron Light Source," Nucl. Instrum. Methods 172、73-76 (May 1980).

24. T. Lauritsen and F. Ajzenberge-Selove. "Energy Levels of Light Nuclei (VII). A $=5-10, "$ Nucl. Phys. 78. $82.97(1966)$.

25. S. Glasstone and M. C. Edlund. "The Slowing Down of Neutrons." in The Elements of Nuciear Reactor Theory (Van Nostrand Press. Princeton. New Jersey. 1952). Chap. 6. 137-190.

26. G. Matone and S. Stipcich, Proc. Workshop un Intermediate Energy Nuclear Physics with Monochromatic and Polarized Photons. Frascati. Italy, July 1980 (Servizio Laboratorio Natinnale de Frascati, 1980).
27. R. Chrien, "A Survey of Research in Neutron Resonance Reactions," Phys. Rep. 64, 334 (September 1980).

28. G. F Auchampaugh, NBS Special Publication 594, Nuclear Cross Sections for Technology, $920-935$ (1980).

29. G. C. Baldwin, J. C. Solem, and V. I. Gol'danskii. "Approaches to the Development of Gamma-Ray Lasers." Rev. Mod. Phys. 53, 687 (October 1981).

30. S. V. Karyagin. "Feasibility of Low-Temperature $\gamma$-Ray Lasers." Sor. Phys. JETP 52. 370 (September 1980).

31. G. C. Baldwin and J. C. Solem. "Maximum Density and Capture Rate of Neutrons Moderated from a Pulsed Source." Nucl. Sci. and Eng. 72. 281 (December 1979). 